\title{
Complete and almost complete minors in double-critical 8-chromatic graphs
}

\author{
Anders Sune Pedersen \\ Dept. of Mathematics and Computer Science \\ University of Southern Denmark \\ Campusvej 55, 5230 Odense M, Denmark \\ asp@imada.sdu.dk \\ Submitted: Jul 30, 2010; Accepted: Mar 18, 2011; Published: Apr 7, 2011 \\ Mathematics Subject Classification: 05C15, 05C83
}

\begin{abstract}
A connected $k$-chromatic graph $G$ is said to be double-critical if for all edges $u v$ of $G$ the graph $G-u-v$ is $(k-2)$-colourable. A longstanding conjecture of Erdös and Lovász states that the complete graphs are the only double-critical graphs. Kawarabayashi, Pedersen and Toft [Electron. J. Combin., 17(1): Research Paper 87,2010 ] proved that every double-critical $k$-chromatic graph with $k \leq 7$ contains a $K_{k}$ minor. It remains unknown whether an arbitrary double-critical 8-chromatic graph contains a $K_{8}$ minor, but in this paper we prove that any double-critical 8-chromatic contains a minor isomorphic to $K_{8}$ with at most one edge missing. In addition, we observe that any double-critical 8-chromatic graph with minimum degree different from 10 and 11 contains a $K_{8}$ minor.
\end{abstract}

\section{Introduction, motivation and main results}

At the very center of the theory of graph colouring is Hadwiger's Conjecture which dates back to 1942 . It states that every $k$-chromatic graph ${ }^{1}$ contains a $K_{k}$ minor.

Conjecture 1.1 (Hadwiger [10]). If $G$ is a $k$-chromatic graph, then $G$ contains a $K_{k}$ minor.

Hadwiger [10] showed that the conjecture holds for $k \leq 4$, the case $k=4$ being the first non-trivial instance of the conjecture. Later, several short and elegant proofs for the case $k=4$ were found; see, for instance, [30]. The case $k=5$ was studied independently by Wagner [31], who proved that the case $k=5$ is equivalent to the Four Colour Problem. In

\footnotetext{
${ }^{1}$ All graphs considered in this paper are undirected, simple, and finite. The reader is referred to Section 2 for basic graph-theoretic terminology and notation.
} 
the early 1960s, Dirac [7] and Wagner [32], independently, proved that every 5-chromatic graph $G$ contains a $K_{5}^{-}$minor. Here $K_{k}^{-}$with $k \in \mathbb{N}$ denotes the complete $k$-graph with one edge missing. The case $k=5$ of Hadwiger's Conjecture was finally settled in the affirmative with Appel and Haken's proof of the Four Colour Theorem [1, 2]. An improved proof was subsequently published in 1997 by Robertson et al. [25]. In 1964, Dirac [8] proved that every 6-chromatic graph contains a $K_{6}^{-}$minor. (See [29, p. 257] for a short version of Dirac's proof.) In 1993, Robertson, Seymour and Thomas [24] proved, using the Four Colour Theorem, that every 6-chromatic graph contains a $K_{6}$ minor. Thus, Hadwiger's Conjecture has been settled in the affirmative for each $k \leq 6$, but remains unsettled for all $k \geq 7$. In the early $1970 \mathrm{~s}$, Jakobsen $[11,12,13]$ proved that for $k=7,8$, and 9 every $k$-chromatic graph contains a minor isomorphic to $K_{7}^{--}, K_{7}^{-}$, and $K_{7}$, respectively, and these results seem to be the best obtained so far in support of Hadwiger's Conjecture for the cases $k=7,8$, and 9 . Here $K_{7}^{--}$denotes a complete 7-graph with two edges missing; there are two non-isomorphic complete 7-graphs with two edges missing. The reader is referred to $[14,30]$ for a thorough survey of Hadwiger's Conjecture and related conjectures.

Another longstanding conjecture in the theory of graph colouring is the so-called Erdős-Lovász Tihany Conjecture which dates back to 1966. This conjecture states, in an interesting special case, that the complete graphs are the only double-critical graphs [9]. A connected $k$-chromatic graph $G$ is double-critical if for all edges $u v$ of $G$ the graph $G-u-v$ is $(k-2)$-colourable.

Conjecture 1.2 (Erdős \& Lovász [9]). If $G$ is a double-critical k-chromatic graph, then $G$ is isomorphic to $K_{k}$.

Conjecture 1.2, which we call the Double-Critical Graph Conjecture, is settled in the affirmative for all $k \leq 5$, but remains unsettled for all $k \geq 6[22,27,28]$. As a relaxed version of the Double-Critical Graph Conjecture the following conjecture was posed in [17].

Conjecture 1.3 (Kawarabayashi et al. [17]). If $G$ is a double-critical $k$-chromatic graph, then $G$ contains a $K_{k}$ minor.

Conjecture 1.3 is, of course, also a relaxed version of Hadwiger's Conjecture, and so we call it the Double-Critical Hadwiger Conjecture; in [17], it was settled in the affirmative for $k \in\{6,7\}$ (without use of the Four Colour Theorem) but it remains open for all $k \geq 8$. Very little seems to be known about complete minors in 8-chromatic graphs. The best result so far in the direction of proving Hadwiger's Conjecture for 8-chromatic graphs seems to be a theorem published in 1970 by Jakobsen [11]; the theorem states that every 8-chromatic graph contains a $K_{7}^{-}$minor. In this paper we prove that every double-critical 8-chromatic graph contains a $K_{8}^{-}$minor. The proof of this result is surprisingly complicated and uses a number of deep results by other authors.

Our main results are as follows.

Theorem 1.4. Every double-critical 8-chromatic graph with minimum degree different from 10 and 11 contains a $K_{8}$ minor. 
Theorem 1.5. Every double-critical 8-chromatic graph contains a $K_{8}^{-}$minor.

The proofs of Theorem 1.4 and Theorem 1.5 are presented in Section 3 and Section 5, respectively. Our proofs do not rely on the Four Colour Theorem but they do rely on the two following deep results.

Theorem 1.6 ((i) Song [26]; (ii) Jørgensen [15]). Suppose $G$ is a graph on at least eight vertices.

(i) If $G$ has more than $\lceil(11 n(G)-35) / 2\rceil$ edges, then $G$ contains a $K_{8}^{-}$minor, and

(ii) if $G$ has more than $6 n(G)-20$ edges, then $G$ contains a $K_{8}$ minor.

\section{Preliminaries and notation}

We shall use standard graph-theoretic terminology and notation as defined in $[4,6]$ with a few additions. Given any graph $G, V(G)$ denotes the vertex set of $G$ and $E(G)$ denotes the edge set of $G$, while $\bar{G}$ denotes the complement of $G$. The order of a graph $G$, that is, the number of vertices in $G$, is denoted $n(G)$, and any graph on $n$ vertices is called an $n$-graph. A vertex of degree $k$ in a graph $G$ is said to be a $k$-vertex (of $G$ ). We write $G \simeq H$ to indicate that the graphs $G$ and $H$ are isomorphic. Given two graphs $H$ and $G$, the complete join of $G$ and $H$, denoted $G+H$, is the graph obtained from two disjoint copies of $H$ and $G$ by joining each vertex of the copy of $G$ to each vertex of the copy of $H$. For every positive integer $k$ and graph $G, k G$ denotes the graph $\sum_{i=1}^{k} G$. Given any edge-transitive graph $G$, any graph, which can be obtained from $G$ by removing one edge, is denoted $G^{-}$. Given any subset $X$ of the vertex set $V(G)$ of a graph $G$, we let $G[X]$ denote the subgraph of $G$ induced by the vertices of $X$. The set of vertices of $G$ adjacent to $v$ is called the neighbourhood of $v$ (in $G$ ), and it is denoted $N_{G}(v)$ or $N(v)$. The set $N(v) \cup\{v\}$ is called the closed neighbourhood of $v$ (in $G$ ), and it is denoted $N_{G}[v]$ or $N[v]$. The induced graph $G[N(v)]$ is referred to as the neighbourhood graph of $v$ with respect to $G$, and it is denoted $G_{v}$. Given two graphs $G$ and $H$, we say that $H$ is a minor of $G$ or that $G$ has an $H$ minor if there is a collection $\left\{V_{h} \mid h \in V(H)\right\}$ of non-empty disjoint subsets of $V(G)$ such that the induced graph $G\left[V_{h}\right]$ is connected for each $h \in V(H)$, and for any two adjacent vertices $h_{1}$ and $h_{2}$ in $H$ there is at least one edge in $G$ joining some vertex of $V_{h_{1}}$ to some vertex of $V_{h_{2}}$. The sets $V_{h}$ are called the branch sets of the minor $H$ of $G$. We may write $H \leq G$ or $G \geq H$, if $G$ contains an $H$ minor. In [17], a number of basic results on double-critical graphs were determined. We will make repeated use of these results and so, for ease of reference, they are restated here.

In the remaining part of this section, we let $G$ denote a non-complete double-critical $k$-chromatic graph with $k \geq 6$. Given any edge $x y \in E(G)$, define

$$
\begin{aligned}
& A(x, y):=N(x) \backslash N[y] \\
& B(x, y):=N(x) \cap N(y) \\
& C(x, y):=N(y) \backslash N[x]
\end{aligned}
$$


Proposition 2.1 ([17]).

(i) $G$ does not contain a complete $(k-1)$-graph as a subgraph,

(ii) $G$ has minimum degree at least $k+1$, and

(iii) for all edges $x y \in E(G)$ and all $(k-2)$-colourings of $G-x-y$, the set $B(x, y)$ of common neighbours of $x$ and $y$ in $G$ contains vertices from every colour class, in particular, $|B(x, y)| \geq k-2$.

Proposition 2.2. If $G[A(x, y)]$ is a complete graph for some edge $x y \in E(G)$, then there is a matching of the vertices of $A(x, y)$ to the vertices of $B(x, y)$ in $\overline{G_{x}}$.

Proof. Suppose $G[A(x, y)]$ is a complete graph for some edge $x y \in E(G)$, and let $G-x-y$ be coloured properly in the colours $1,2, \ldots, k-3$, and $k-2$. The colours applied to $A(x, y)$ are all distinct, and so we may assume $A(x, y)=\left\{a_{1}, \ldots, a_{p}\right\}$ where vertex $a_{i}$ is coloured $i$ for each $a_{i} \in A(x, y)$. According to Proposition 2.1 (iii), each of the colours $1,2, \ldots, k-3$, and $k-2$ appear at least once on a vertex of $B(x, y)$, say $B(x, y)=\left\{b_{1}, \ldots, b_{q}\right\}$ with vertex $b_{i}$ being coloured $i$ for each $i \leq k-2$. Now $\left\{a_{1} b_{1}, a_{2} b_{2}, \ldots, a_{p} b_{p}\right\}$ is a matching of the vertices of $A(x, y)$ to vertices of $B(x, y)$ in $\overline{G_{x}}$.

Proposition 2.3 ([17]). If the set $A(x, y)$ is non-empty for some edge $x y \in E(G)$, then $\delta(G[A(x, y)]) \geq 1$, that is, the induced subgraph $G[A(x, y)]$ contains no isolated vertices. By symmetry, $\delta(G[C(x, y)]) \geq 1$, if $C(x, y)$ is non-empty.

Thus, by Proposition 2.3, if $y$ is a vertex which has degree 2 in $\overline{G_{x}}$ then the two neighbours of $y$ in $\overline{G_{x}}$ must be non-adjacent in $\overline{G_{x}}$. In particular, no component of $\overline{G_{x}}$ is a triangle.

Proposition $2.4([17])$.

(i) For any vertex $x$ of $G$ not joined to all other vertices of $G, \chi\left(G_{x}\right) \leq k-3$;

(ii) if $x$ is a vertex of degree $k+1$ in $G$, then the complement $\overline{G_{x}}$ consists of isolated vertices (possibly none) and cycles (at least one), where the length of each cycle is at least 5 , and

(iii) $G$ is 6-connected.

\section{Minimum degree 9 and $K_{8}$ minors}

Proposition 3.1. If $G$ is a double-critical 8-chromatic graph with a vertex $x$ of degree 9, then $G_{x} \simeq \overline{C_{8}}+K_{1}$ or $G_{x} \simeq \overline{C_{9}}$. 
Proof. Suppose $G$ is a double-critical 8-chromatic graph with a vertex $x$ of degree 9 . Now, according to Proposition 2.4 (ii), $G_{x}$ consists of isolated vertices and cycles (at least one cycle) of length at least 5 . Since $G_{x}$ consists of only nine vertices, it follows that $\overline{G_{x}}$ consists of exactly one cycle, whose length we denote by $j$, and some isolated vertices. If $j \in\{5,6\}$, then $G[N[x]]$ is easily seen to contain $K_{7}$ as a subgraph, contrary to Proposition 2.1 (i). Suppose $j=7$. Moreover, suppose that the vertex $x$ is not adjacent to all other vertices of $G$. Then, according to Proposition 2.4 (i), $\chi\left(G_{x}\right) \leq 5$. However, the graph $G_{x}$, which is isomorphic to $\overline{C_{7}}+K_{2}$, is easily seen not be 5 -colourable. Thus, the vertex $x$ is adjacent to all other vertices of $G$, and so $G$ is isomorphic to $\overline{C_{7}}+K_{3}$. However, the graph $\overline{C_{7}}+K_{3}$ is easily seen to be 7 -colourable, a contradiction. Thus, we must have $j \geq 8$, and so the desired result follows immediately.

Proposition 3.1 implies that any double-critical 8-chromatic graph with a vertex of degree 9 contains $K_{6}^{-}$as a subgraph.

Proposition 3.2. Every double-critical 8-chromatic graph with minimum degree 9 contains a $K_{8}$ minor.

Proof. Suppose $G$ is a double-critical 8-chromatic graph with minimum degree 9, and let $x$ denote a vertex of $G$ of degree 9. As in the proof of Proposition 3.1, it follows that $G-N[x]$ contains at least one vertex. Let $z$ denote a vertex of $G-N[x]$. According to Proposition 3.1, there are two cases to consider: either $G_{x} \simeq \overline{C_{8}}+K_{1}$ or $G_{x} \simeq \overline{C_{9}}$. It is easy to find a $K_{7}$ minor in $\overline{C_{8}}+K_{1}$, and so we need only consider the case where $G_{x}$ is isomorphic to $\overline{C_{9}}$. Let the vertices of the 9 -cycle $\overline{G_{x}}$ be labelled cyclically $v_{0} v_{1} v_{2} \ldots v_{8}$. By Proposition 2.4 (iii), $G$ is 6-connected. Now, according to Menger's Theorem (see, for instance, [4, Theorem 9.1]), there is a collection $\mathcal{C}$ of six internally vertex-disjoint $(x, z)$-paths in $G$. Obviously, each path $P \in \mathcal{C}$ contains a vertex from $V\left(G_{x}\right)$, and we may assume that each of the paths $P \in \mathcal{C}$ contains exactly one vertex from $V\left(G_{x}\right)$. The fact that there are nine vertices in $V\left(G_{x}\right)$ and six vertex-disjoint $(x, z)$-paths in $\mathcal{C}$ going through $V\left(G_{x}\right)$ implies the existence of a pair of vertices $v_{i}$ and $v_{i+1}$ (modulo 9) such that there are $(x, z)$-paths $Q_{i}, Q_{i+1} \in \mathcal{C}$ going through $v_{i}$ and $v_{i+1}$, respectively. We may, by symmetry, assume $i=0$. We contract the $\left(v_{0}, v_{1}\right)$-path $\left(Q_{0} \cup Q_{1}\right)-x$ in $G$ to an

edge between $v_{0}$ and $v_{1}$. The resulting graph contains the graph $H:=\overline{C_{9}^{-}}+K_{1}$ as a subgraph (here $C_{9}=v_{0} v_{1} \ldots v_{8}$ and $v_{0} v_{1}$ is the 'missing edge' of $C_{9}^{-}$). The graph $H$ can be contracted to $K_{8}$ by contracting the edges $v_{2} v_{6}$ and $v_{4} v_{8}$. Thus, $G \geq K_{8}$, as desired.

Proof of Theorem 1.4. Suppose $G$ is a non-complete double-critical 8-chromatic graph. Then, according to Proposition 2.1 (ii), $\delta(G) \geq 9$. If $\delta(G) \geq 12$, then $|E(G)| \geq 6 n(G)$ and so, by Theorem 1.6 (ii), $G \geq K_{8}$. If $\delta(G)=9$, then the desired result follows from Proposition 3.2.

\section{Minimum degree 10 and $K_{8}$ minors}

Observation 4.1. If $G$ is a double-critical 8-chromatic graph with minimum degree 10, then $\Delta\left(\overline{G_{x}}\right) \leq 3$ for every vertex $x \in V(G)$ with $\operatorname{deg}(x, G)=10$. 
Proof. Suppose $G$ is a double-critical 8-chromatic graph with minimum degree 10 and $\Delta\left(\overline{G_{x}}\right) \geq 4$. Let $y$ denote a vertex which has degree at least 4 in $\overline{G_{x}}$. Then $|A(x, y)| \geq 4$ and, according to Proposition 2.1 (iii), $|B(x, y)| \geq 6$. Thus, $\operatorname{deg}(x, G) \geq|A(x, y)|+$ $|B(x, y)|+1 \geq 11$, which contradicts the assumption $\operatorname{deg}(x, G)=10$.

Proposition 4.2. Suppose $G$ is a double-critical 8-chromatic graph with minimum degree 10 , and suppose $G$ contains a vertex $x$ of degree 10 such that $\Delta\left(\overline{G_{x}}\right) \leq 2$. Then $G$ contains a $K_{8}$ minor.

Proof. Suppose $G$ is a double-critical 8-chromatic graph with minimum degree 10, and suppose $G$ contains a vertex $x$ of degree 10 such that $\Delta\left(\overline{G_{x}}\right) \leq 2$.

If $\Delta\left(\overline{G_{x}}\right)=0$, then $G_{x} \simeq K_{10}$, a contradiction. According to Proposition 2.3, no

vertex of $\overline{G_{x}}$ has degree exactly 1 . Hence, $\Delta\left(\overline{G_{x}}\right)=2$, and so the graph $\overline{G_{x}}$ consists of cycles (at least one) and possibly some isolated vertices. According to the remark after Proposition 2.3, the cycles of $\overline{G_{x}}$ all have length at least 4 . If $\overline{G_{x}}$ has at least five isolated vertices, then it is easy to see that $G_{x}$ contains $K_{7}$ as a subgraph. If $\overline{G_{x}}$ has exactly four isolated vertices then $G_{x} \simeq K_{4}+\overline{C_{6}}$ and $G_{x} \supset K_{7}$. If $\overline{G_{x}}$ has exactly three isolated vertices, then $G_{x} \simeq K_{3}+\overline{C_{7}}$. If $\overline{G_{x}}$ has exactly two isolated vertices, then $G_{x}$ is isomorphic to either $K_{2}+2 \overline{C_{4}}$, or $K_{2}+\overline{C_{8}}$. If $\overline{G_{x}}$ has exactly one isolated vertex, then $G_{x}$ is isomorphic to either $K_{1}+\overline{C_{4}}+\overline{C_{5}}$, or $K_{1}+\overline{C_{9}}$. If $\overline{G_{x}}$ has no isolated vertices, then $G_{x}$ is isomorphic to either $\overline{C_{4}}+\overline{C_{6}}, 2 \overline{C_{5}}$, or $\overline{C_{10}}$. In each case it is easy to exhibit a $K_{7}$ minor in $G_{x}$, and so $G \geq K_{8}$.

\section{$5 \quad$ Minimum degree 10 and $K_{8}^{-}$minors}

In this section, we shall apply the following result of Mader.

Theorem 5.1 (Mader [19]). Every graph with minimum degree at least 5 contains $K_{6}^{-}$ or the icosahedral graph as a minor. In particular, every graph with minimum degree at least 5 and at most 11 vertices contains a $K_{6}^{-}$minor.

A proof of Theorem 5.1 may also be found in [3, p. 373].

Proposition 5.2. Suppose $G$ is a double-critical 8-chromatic graph with minimum degree 10. If $G$ contains a vertex $x$ of degree 10 such that $G_{x}$ contains at least one vertex of degree 9 in $G_{x}$, then $G$ contains a $K_{8}^{-}$minor.

Proof. Suppose $G$ is a double-critical 8-chromatic graph with minimum degree 10 and a vertex $x \in V(G)$ with $\operatorname{deg}(x, G)=10$ such that a vertex of $V\left(G_{x}\right)$, say $v$, has degree 9 in $G_{x}$. According to Observation $4.1, \Delta\left(\overline{G_{x}}\right) \leq 3$ and so $\delta\left(G_{x}\right)=n\left(G_{x}\right)-1-\Delta\left(\overline{G_{x}}\right) \geq 6$. Thus, the graph $G_{x}-v$ has minimum degree at least 5 and exactly 9 vertices, and so it follows from Theorem 5.1 that $G_{x}-v$ contains a $K_{6}^{-}$minor. Such a $K_{6}^{-}$minor of $G_{x}-v$ along with the additional branch sets $\{x\}$ and $\{v\}$ constitute a $K_{8}^{-}$minor of $G$. 
Lemma 5.3. Suppose $G$ is a graph with a vertex $x$ of degree 10 such that $\overline{G_{x}}$ is connected and cubic. Suppose, in addition, that there is a vertex $z \in V(G) \backslash N_{G}[x]$ such that $G$ contains at least six internally vertex-disjoint $(x, z)$-paths. Then $G$ contains a $K_{8}^{-}$minor.

Proof. Suppose $G$ is a graph with a vertex $x$ of degree 10 such that $\overline{G_{x}}$ is connected and cubic. Suppose, in addition, that there is a vertex $z \in V(G) \backslash N_{G}[x]$ such that $G$ contains at least six internally vertex-disjoint $(x, z)$-paths.

There are exactly 21 non-isomorphic cubic graphs of order 10, see, for instance, [23]. These 21 non-isomorphic cubic graphs of order 10 are depicted in Appendix A; let these graphs be denoted as in Appendix A. If $\overline{G_{x}} \simeq G_{i}$, where $i \in[19] \backslash\{7,8,9,12,17\}$, then the labelling of the vertices of the graph $G_{i}$ indicates how $\overline{G_{i}}$ may be contracted to $K_{7}^{-}$ or $K_{7}$. The vertices labelled $j \in[7]$ constitute the $j$ th branch set of a $K_{7}^{-}$minor or $K_{7}$ minor. If the branch sets only constitute a $K_{7}^{-}$minor, then it is because there is no edge between the branch sets of vertices labelled 1 and 7 . In these cases we obtain $G \geq K_{8}^{-}$. In

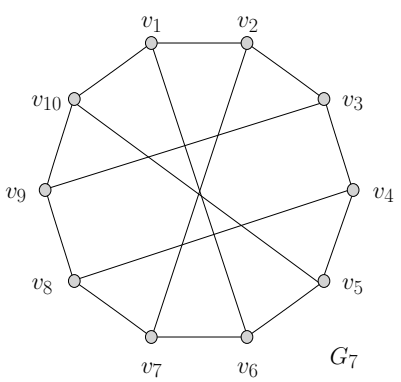

(a) The graph $G_{7}$.

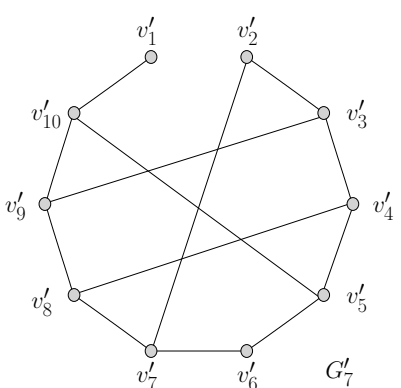

(b) The graph $G_{7}^{\prime}$.

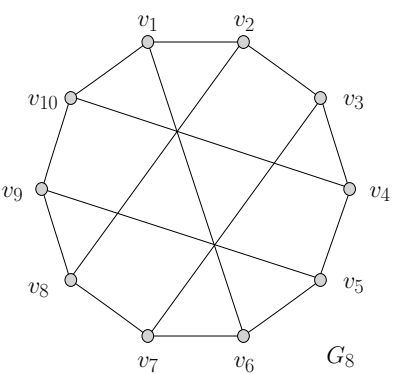

(c) The graph $G_{8}$.

Figure 1: The graphs $G_{7}, G_{7}^{\prime}$, and $G_{8}$, which occur in the cases (i) and (ii) in the proof of Lemma 5.3.

order to handle the cases $\overline{G_{x}} \simeq G_{i}$, where $i \in\{7,8,9,12,17\}$, we use the assumption that $V(G) \backslash N_{G}[x]$ contains a vertex $z$ such that $G$ has a collection $\mathcal{R}$ of at least six internally vertex-disjoint $(x, z)$-paths. We may assume that each of the paths in $\mathcal{R}$ contains exactly one vertex from $V\left(G_{x}\right)$.

(i) Suppose $\overline{G_{x}} \simeq G_{7}$ with the vertices of $\overline{G_{x}}$ labelled as shown in Figure 1 (a). Let $\mathcal{S}$ denote the collection of the five 2-sets $\left\{v_{1}, v_{6}\right\},\left\{v_{2}, v_{7}\right\},\left\{v_{3}, v_{9}\right\},\left\{v_{4}, v_{8}\right\}$, and $\left\{v_{5}, v_{10}\right\}$. Since the 2 -sets in $\mathcal{S}$ are pairwise disjoint and cover $N_{G}(x)$, it follows from the pigeonhole principle that at least two of the internally vertex-disjoint $(x, z)$ paths, say $Q_{1}$ and $Q_{2}$, of $\mathcal{R}$ go through the same 2-set $S:=\left\{v_{i}, v_{j}\right\} \in \mathcal{S}$.

Suppose $S \in \mathcal{S} \backslash\left\{\left\{v_{1}, v_{6}\right\}\right\}$. By symmetry, we may assume $S \in\left\{\left\{v_{2}, v_{7}\right\},\left\{v_{3}, v_{9}\right\}\right\}$. In each case we contract the $\left(v_{i}, v_{j}\right)$-path $\left(Q_{1} \cup Q_{2}\right)-x$ into the edge $v_{i} v_{j}$ and obtain a graph which has a $K_{7}^{-}$minor in the neighbourhood of $x$ : If $S=\left\{v_{2}, v_{7}\right\}$, then the branch sets $\left\{v_{1}, v_{4}\right\},\left\{v_{2}, v_{7}\right\},\left\{v_{3}\right\},\left\{v_{5}\right\},\left\{v_{6}, v_{9}\right\},\left\{v_{8}\right\}$, and $\left\{v_{10}\right\}$ form a $K_{7}^{-}$ minor in $G_{x}$. If $S=\left\{v_{3}, v_{9}\right\}$, then the branch sets $\left\{v_{1}, v_{8}\right\},\left\{v_{2}, v_{10}\right\},\left\{v_{3}\right\},\left\{v_{4}, v_{6}\right\}$, 
$\left\{v_{5}\right\},\left\{v_{7}\right\}$, and $\left\{v_{9}\right\}$ form a $K_{7}^{-}$minor in $G_{x}$. In both cases we obtain $G \geq K_{8}^{-}$, as desired.

Hence, we may assume $S=\left\{v_{1}, v_{6}\right\}$ and that $\mathcal{R}$ contains no such two paths going through the same 2-set of $\mathcal{S} \backslash\left\{\left\{v_{1}, v_{6}\right\}\right\}$. Since $|\mathcal{R}| \geq 6$, there is precisely one path going through each of the sets $S^{\prime} \in \mathcal{S} \backslash\left\{\left\{v_{1}, v_{6}\right\}\right\}$. By symmetry of $\overline{G_{x}}$, we may assume that there is an $(x, z)$-path $Q_{3} \in \mathcal{R}$ going through the vertex $v_{2}$ of $N_{G}(x)$. Now, by contracting the $\left(v_{2}, z\right)$-path $Q_{3}-x$ and the $\left(v_{6}, z\right)$-path $Q_{2}-x$ into two edges, and then contracting the $\left(v_{1}, z\right)$-path $Q_{1}-x$ into one vertex, we obtain a graph $G^{\prime}$ in which the neighbourhood graph $G_{x}^{\prime}$ of $x$ contains the complement of the $G_{7}^{\prime}$, depicted in Figure 1 (b), as a subgraph. The branch sets $\left\{v_{1}^{\prime}\right\},\left\{v_{2}^{\prime}\right\},\left\{v_{3}^{\prime}, v_{5}^{\prime}\right\}$, $\left\{v_{4}^{\prime}, v_{9}^{\prime}\right\},\left\{v_{6}^{\prime}\right\},\left\{v_{7}^{\prime}, v_{10}^{\prime}\right\}$, and $\left\{v_{8}^{\prime}\right\}$ constitute a $K_{7}^{-}$minor in $\overline{G_{7}^{\prime}}$, and so $G \geq K_{8}^{-}$.

(ii) Suppose $\overline{G_{x}} \simeq G_{8}$ with the vertices of $\overline{G_{x}}$ labelled as shown in Figure 1 (c). In this case we contract a path $(P \cup Q)-x$ with $P, Q \in \mathcal{R}$ into an edge $e \in$ $\left\{v_{1} v_{6}, v_{2} v_{8}, v_{3} v_{7}, v_{4} v_{10}, v_{5} v_{9}\right\}$ which is missing in $G_{x}$. By the symmetry of $G_{x}$, we need only consider the cases $e=v_{1} v_{6}$ and $e=v_{2} v_{8}$. If $e=v_{1} v_{6}$, then the branch sets $\left\{v_{1}, v_{5}\right\},\left\{v_{2}\right\},\left\{v_{3}, v_{9}\right\},\left\{v_{4}, v_{7}\right\},\left\{v_{6}\right\},\left\{v_{8}\right\}$, and $\left\{v_{10}\right\}$ constitute a $K_{7}^{-}$minor in the neighbourhood of $x$. If $e=v_{2} v_{8}$, then the branch sets $\left\{v_{1}, v_{9}\right\},\left\{v_{2}\right\},\left\{v_{3}, v_{6}\right\}$, $\left\{v_{4}, v_{7}\right\},\left\{v_{5}\right\},\left\{v_{8}\right\}$, and $\left\{v_{10}\right\}$ constitute a $K_{7}^{-}$minor in the neighbourhood of $x$. In both cases we obtain $G \geq K_{8}^{-}$.

(iii) Suppose $\overline{G_{x}} \simeq G_{9}$ with the vertices of $\overline{G_{x}}$ labelled as shown in Figure 2 (a). As in case (ii), we contract a path $(P \cup Q)-x$ with $P, Q \in \mathcal{R}$ into an edge $e \in\left\{v_{1} v_{6}, v_{2} v_{10}, v_{3} v_{7}, v_{4} v_{8}, v_{5} v_{9}\right\}$. By the symmetry of $G_{x}$, we need only consider $e \in\left\{v_{1} v_{6}, v_{2} v_{10}, v_{3} v_{7}, v_{4} v_{8}\right\}$. If $e=v_{1} v_{6}$, then the branch sets $\left\{v_{1}\right\},\left\{v_{2}, v_{5}\right\},\left\{v_{3}\right\}$, $\left\{v_{4}, v_{9}\right\},\left\{v_{6}\right\},\left\{v_{7}, v_{10}\right\}$, and $\left\{v_{8}\right\}$ constitute a $K_{7}^{-}$minor in the neighbourhood of $x$. If $e=v_{2} v_{10}$, then the branch sets $\left\{v_{1}, v_{8}\right\},\left\{v_{2}\right\},\left\{v_{3}, v_{5}\right\},\left\{v_{4}\right\},\left\{v_{6}, v_{9}\right\}\left\{v_{7}\right\}$, and $\left\{v_{10}\right\}$ constitute a $K_{7}^{-}$minor in the neighbourhood of $x$. If $e=v_{3} v_{7}$, then the branch sets $\left\{v_{1}, v_{8}\right\},\left\{v_{2}, v_{6}\right\},\left\{v_{3}\right\},\left\{v_{4}, v_{10}\right\},\left\{v_{5}\right\},\left\{v_{7}\right\}$, and $\left\{v_{9}\right\}$ constitute a $K_{7}^{-}$ minor in the neighbourhood of $x$. If $e=v_{4} v_{8}$, then the branch sets $\left\{v_{1}\right\},\left\{v_{2}, v_{5}\right\}$, $\left\{v_{3}, v_{9}\right\},\left\{v_{4}\right\},\left\{v_{6}\right\},\left\{v_{7}, v_{10}\right\}$, and $\left\{v_{8}\right\}$ constitute a $K_{7}^{-}$minor in the neighbourhood of $x$. In each case we obtain $G \geq K_{8}^{-}$.

(iv) Suppose $\overline{G_{x}} \simeq G_{12}$ with the vertices of $\overline{G_{x}}$ labelled as in Figure 2 (b). Again, we contract a path $(P \cup Q)-x$ with $P, Q \in \mathcal{R}$ into an edge $e \in\left\{v_{1} v_{6}, v_{2} v_{4}, v_{3} v_{7}, v_{5} v_{9}, v_{8} v_{10}\right\}$. By the symmetry of $G_{x}$, we need only consider the cases $e \in\left\{v_{1} v_{6}, v_{2} v_{4}, v_{3} v_{7}\right\}$. If $e=v_{1} v_{6}$, then the branch sets $\left\{v_{1}\right\},\left\{v_{2}, v_{7}\right\},\left\{v_{3}, v_{9}\right\},\left\{v_{4}, v_{10}\right\},\left\{v_{5}\right\},\left\{v_{6}\right\}$, and $\left\{v_{8}\right\}$ constitute a $K_{7}^{-}$minor in the neighbourhood of $x$. If $e=v_{2} v_{4}$, then the branch sets $\left\{v_{1}, v_{5}\right\},\left\{v_{2}\right\},\left\{v_{3}, v_{8}\right\},\left\{v_{4}\right\},\left\{v_{6}\right\},\left\{v_{7}, v_{10}\right\}$, and $\left\{v_{9}\right\}$ constitute a $K_{7}^{-}$minor in the neighbourhood of $x$. If $e=v_{3} v_{7}$, then the branch sets $\left\{v_{1}, v_{9}\right\},\left\{v_{2}, v_{6}\right\},\left\{v_{3}\right\}$, $\left\{v_{4}, v_{8}\right\},\left\{v_{5}\right\},\left\{v_{7}\right\}$, and $\left\{v_{10}\right\}$ constitute a $K_{7}^{-}$minor in the neighbourhood of $x$. In each case we obtain $G \geq K_{8}^{-}$.

(v) Suppose $\overline{G_{x}} \simeq G_{17}$ with the vertices of $\overline{G_{x}}$ labelled as shown in Figure 2 (c). The graph $G_{17}$ is the Petersen graph, and the complement of the Petersen graph does 
not contain a $K_{7}$ minor. However, we may repeat the trick used in the previous cases to obtain a $K_{7}^{-}$minor. We contract a path $(P \cup Q)-x$ with $P, Q \in \mathcal{R}$, into an edge $e \in\left\{v_{i} v_{i+5} \mid i \in[5]\right\}$. By the symmetry of $G_{x}$, we may assume $e=v_{1} v_{6}$. Now, the branch sets $\left\{v_{1}\right\},\left\{v_{2}, v_{8}\right\},\left\{v_{3}\right\},\left\{v_{4}, v_{10}\right\},\left\{v_{5}, v_{9}\right\},\left\{v_{6}\right\}$, and $\left\{v_{7}\right\}$ constitute a $K_{7}^{-}$minor in the neighbourhood of $x$. Thus, $G$ contains a $K_{8}^{-}$minor.

This completes the proof.

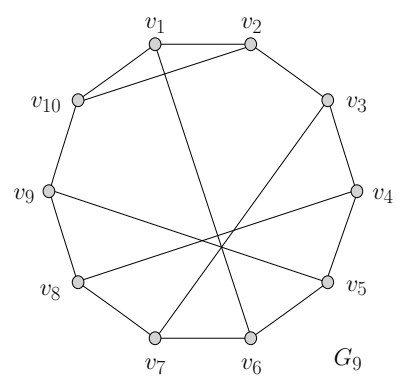

(a) The graph $G_{9}$.

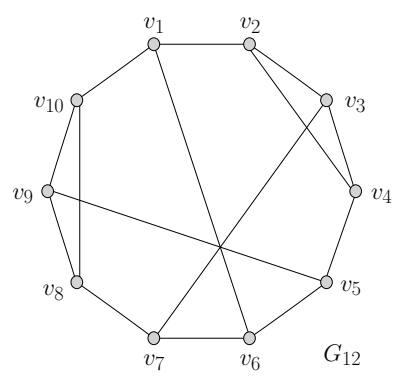

(b) The graph $G_{12}$.

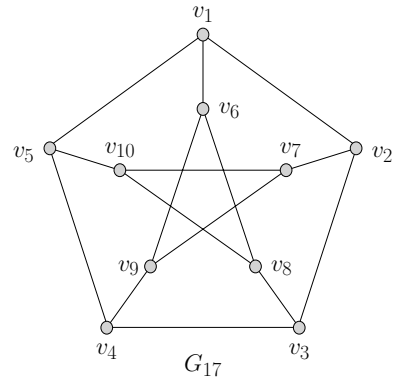

(c) The graph $G_{17}$.

Figure 2: The graphs $G_{9}, G_{12}$, and $G_{17}$, which occur in the cases (iii), (iv), and (v) in the proof of Lemma 5.3.

Proposition 5.4. Suppose $G$ is a double-critical 8-chromatic graph with minimum degree 10. If $G$ contains a vertex $x$ of degree 10 such that $G_{x}$ contains no vertex of degree 9 in $G_{x}$, then $G$ contains a $K_{8}^{-}$minor.

Proof. Suppose $G$ is a double-critical 8-chromatic graph with minimum degree 10, and suppose $G$ contains a vertex $x$ of degree 10 such that $G_{x}$ contains no vertex of degree 9 in $G_{x}$. Then it follows from Proposition 2.3 and Observation 4.1 that each vertex of $\overline{G_{x}}$ has degree 2 or 3.

We first consider the case where $\overline{G_{x}}$ is disconnected. Since $\delta\left(\overline{G_{x}}\right) \geq 2$, it follows that any component of $\overline{G_{x}}$ contains at least three vertices. If $\overline{G_{x}}$ contains a component on three vertices, then this component is a $K_{3}$; this contradicts Proposition 2.3. Hence, each component of $\overline{G_{x}}$ contains at least four vertices, and so, since $n\left(G_{x}\right)=10$, it follows that $\overline{G_{x}}$ contains precisely two components, say $D_{1}$ and $D_{2}$ with $n\left(D_{1}\right) \leq n\left(D_{2}\right)$. Suppose $n\left(D_{1}\right)=4$. The fact that $\delta\left(\overline{G_{x}}\right) \geq 2$ implies that $D_{1}$ must contain a 4 -cycle, and so it is easy to see that $D_{1}$ must be $C_{4}, K_{4}^{-}$, or $K_{4}$. This, however, contradicts Proposition 2.2 or Proposition 2.3, and so we must have $n\left(D_{1}\right)=n\left(D_{2}\right)=5$. Of course, if $G^{\prime}$ is a subgraph of $G$, and $G^{\prime}$ contains an $H$ minor, then $G$ contains an $H$ minor. Thus, it suffices to consider the case where both $D_{1}$ and $D_{2}$ contain exactly one vertex of degree 2 , in which case both $D_{1}$ and $D_{2}$ are isomorphic to $K_{4}$ with exactly one edge subdivided. In this case it is very easy to find a $K_{7}$ minor in $G_{x}$.

Suppose that $\overline{G_{x}}$ is connected, and let $D$ denote $\overline{G_{x}}$. If $x$ is adjacent to all other vertices of $G$, then $n(G)=11$ and so, since $\delta(G)=10, G \simeq K_{11}$, a contradiction. Thus, 
$V(G) \backslash N_{G}[x]$ is non-empty. Let $z$ denote a vertex of $V(G) \backslash N_{G}[x]$. By Proposition 2.4 (iii) and Menger's Theorem, there are six internally vertex-disjoint $(x, z)$-paths in $G$. If $D$ is cubic, then, according to Lemma $5.3, G \geq K_{8}^{-}$. Suppose that $D$ is not cubic. We add edges (possibly none) between pairs of non-adjacent 2-vertices of $D$ to obtain a subcubic graph $D^{\prime}$, which contains no pair of non-adjacent 2-vertices. Suppose $D^{\prime}$ is cubic. Then $G^{\prime}:=G \backslash\left(E\left(D^{\prime}\right) \backslash E(D)\right)$ satisfies the assumption of Lemma 5.3, that is, $D^{\prime}$ is a connected cubic 10-graph; $G^{\prime}$ has six internally vertex-disjoint $(x, z)$-paths, since $G$ has six internally vertex-disjoint $(x, z)$-paths, and these may be chosen so that they do not contain any edge of $E\left(G_{x}\right)$. Thus, by Lemma $5.3, G^{\prime} \geq K_{8}^{-}$, which implies that the supergraph $G$ of $G^{\prime}$ has a $K_{8}^{-}$minor.

Now, suppose $D^{\prime}$ is not cubic. Then $D^{\prime}$ is a connected subcubic 10-graph with minimum degree 2. Thus, since the number of odd degree vertices of any graph is even, it follows that $D^{\prime}$ contains at least two 2-vertices. Recall, that $D^{\prime}$ contains no pair of non-adjacent 2 -vertices. This means that that $D^{\prime}$ must contain exactly two 2 -vertices and that these must be neighbours. There are exactly 23 connected 10 -graphs each with two 2-vertices and eight 3 -vertices, where the two 2-vertices are adjacent. ${ }^{2}$ These graphs, denoted $J_{i}(i \in[23])$, are depicted in Appendix B. For each $i \in[23]$, the labelling of the vertices of the graph $J_{i}$ indicates how $\overline{J_{i}}$ may be contracted to $K_{7}^{-}$or, even, $K_{7}$; the vertices labelled $j \in[7]$ constitute the $j$ th branch set of a $K_{7}^{-}$minor or $K_{7}$ minor. If the branch sets only constitute a $K_{7}^{-}$minor, then it is because there is no edge between the branch sets labelled 1 and 7 . This completes the proof.

Proof of Theorem 1.5. Let $G$ denote a double-critical 8-chromatic graph. By Theorem 1.4, we may assume $\delta(G) \in\{10,11\}$. If $\delta(G)=11$, then $|E(G)| \geq 11 n(G) / 2$ and so, by Theorem 1.6 (i), $G \geq K_{8}^{-}$. Suppose $\delta(G)=10$, and let $x$ denote a vertex of degree 10 in $G$. If $G_{x}$ contains at least one vertex of degree 9 , then the desired conclusion follows from Proposition 5.2. On the other hand, if $G_{x}$ contains no vertex of degree 9, then the desired conclusion follows from Proposition 5.4. This completes the proof.

\section{More open problems}

The Double-Critical Graph Conjecture is still open for 6-chromatic graphs. To settle this instance of the conjecture in the affirmative, it would, by Proposition 2.1 (i), suffice to prove that any double-critical 6-chromatic graph contains $K_{5}$ as a subgraph; however, we cannot even prove that such a graph contains $K_{4}$ as a subgraph.

Problem 6.1 (Matthias Kriesell ${ }^{3}$ ). Prove that every double-critical 6-chromatic graph contains $K_{4}$ as a subgraph.

\footnotetext{
${ }^{2}$ According to the computer program geng developed by Brendan McKay [21], there are 113 connected graphs of order 10 each with two 2-vertices and eight 3-vertices - among these graphs exactly 23 have the property that the two 2-vertices are adjacent. This latter fact was verified, independently, by inspection by the author and by a computer program developed by Marco Chiarandini.

${ }^{3}$ Private communication to the author, Odense, September, 2008.
} 


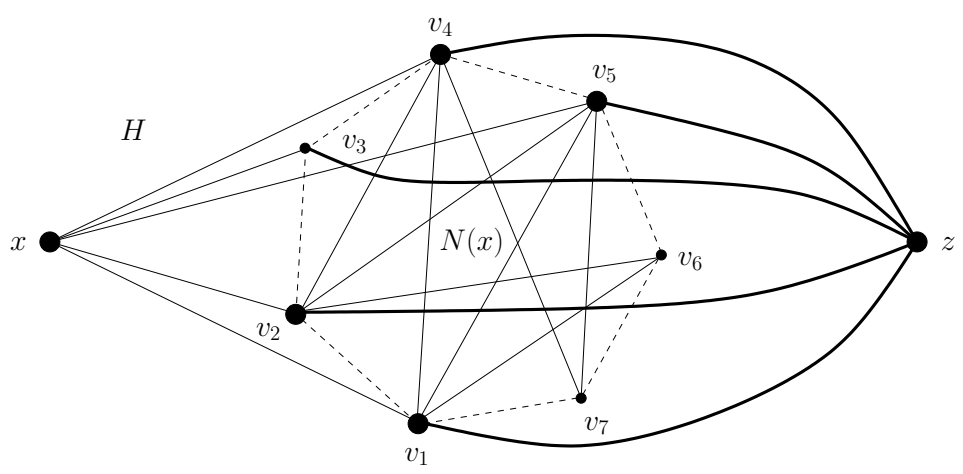

Figure 3: The subgraph $H$ of $G$ is a subdivision of $K_{6}$. The six larger dots represent the branch vertices of $H$, while the smaller dots represent subdividing vertices. The filled straight lines represent edges in $H$, while the bold curves represent the paths $Q_{1}-x$, $Q_{2}-x, Q_{3}-x, Q_{4}-x$, and $Q_{5}-x$.

In [17], it was proved that every double-critical 6-chromatic graph contains a $K_{6}$ minor. A stronger result would be that every double-critical 6-chromatic graph contains a subdivision of $K_{6}$.

Problem 6.2. Prove that every double-critical 6-chromatic graph $G$ contains a subdivision of $K_{6}$.

According to Observation 6.3, Problem 6.2 has a positive solution if $G$ has minimum degree at most 7 .

Mader [20] proved a longstanding conjecture, known as Dirac's Conjecture, which states that any graph $G$ with at least three vertices and at least $3 n(G)-5$ edges contains a subdivision of $K_{5}$. Thus, in particular, every double-critical 6-chromatic graph contains a subdivision of $K_{5}$. Here we prove that every double-critical 6-chromatic graph with minimum degree at most 7 contains a subdivision of $K_{6}$.

Observation 6.3. Any double-critical 6-chromatic graph with minimum degree at most 7 contains a subdivision of $K_{6}$.

Proof of Observation 6.3. Let $G$ denote any double-critical 6-chromatic graph with minimum degree at most 7. If $\delta(G) \leq 6$, then, by Proposition 2.1 (ii), $G \simeq K_{6}$. Hence $\delta(G)=7$. Let $x$ denote a vertex of degree 7 in $G$. If $x$ is adjacent to all other vertices of $G$, then, since $\delta(G)=7, G \simeq K_{8}$, a contradiction. Hence $G-N[x]$ is non-empty. Let $z$ denote a vertex of $G-N[x]$. According to Corollary 6.1 in [17], $\overline{G_{x}}$ is a 7-cycle $C_{7}$ with, say, $C_{7}=v_{1} v_{2} v_{3} \ldots v_{7}$. By Proposition 2.4 (iii), $G$ is 6-connected, and so there is a collection $\mathcal{C}=\left\{Q_{1}, Q_{2}, \ldots, Q_{6}\right\}$ of six internally vertex $(x, z)$-paths in $G$. We may assume that each path $Q_{i} \in \mathcal{C}$ contains exactly one vertex from $V\left(G_{x}\right)$. By the symmetry of $G_{x}$, we may, without loss of generality, assume that $V\left(Q_{i}\right) \cap V\left(G_{x}\right)=\left\{v_{i}\right\}$ for each $i \in[6]$. Thus, in $G$, there is a $K_{6}$-subdivision $H$ with branch vertices $v_{1}, v_{2}, v_{4}, v_{5}, x$, and $z$. The paths in $H$ connecting the branch vertices of $K_{6}$ are as indicated in Figure 3. Thus, $G$ contains a subdivision of $K_{6}$. 
The following conjecture, known as the $(k-1,1)$ Minor Conjecture, is a relaxed version of Hadwiger's Conjecture.

Conjecture 6.4 (Chartrand, Geller \& Hedetniemi [5]; Woodall [33]). Every k-chromatic graph has either a $K_{k}$ minor or a $K_{\left\lfloor\frac{k+1}{2}\right\rfloor,\left\lceil\frac{k+1}{2}\right\rceil}$ minor.

Kawarabayashi and Toft [16] proved that every 7-chromatic graph contains $K_{7}$ or $K_{4,4}$ as a minor - thus, settling the case $k=7$ of the $(k-1,1)$ Minor Conjecture. This result has inspired the following problem.

Problem 6.5. Prove that every double-critical 8-chromatic graph contains $K_{8}$ or $K_{4,5}$ as a minor.

A natural generalisation of Problem 6.1 would be to ask for a linear function $f$ such that every double-critical $k$-chromatic graph has a clique of order $f(k)$; if that problem is too hard it might be worth considering the following problem.

Problem 6.6 (Sergey Norin $\left.{ }^{4}\right)$. Prove that there is a linear strictly increasing function $f$ such that every double-critical $k$-chromatic graph has a complete minor of order $f(k)$.

\section{Acknowledgement}

I thank Marco Chiarandini, Daniel Merkle, Friedrich Regen and Bjarne Toft for stimulating discussions on critical graphs and for assistance in using certain programs, in particular, I thank Friedrich and Marco for developing programs for sorting and displaying small graphs. Moreover, I thank two anonymous referees for their valuable comments and suggestions, which helped to improve this paper.

\footnotetext{
${ }^{4}$ Private communication to the author at Prague Midsummer Combinatorial Workshop XV, July 27 - July 31, 2009.
} 


\section{Appendix A}

This section contains drawings of all non-isomorphic cubic graphs $G_{i}(i \in[21])$ of order 10. The drawings are copies of drawings found in [18]. Drawings of all non-isomorphic cubic graphs of order at most 14 can be found in [23].

For $i \in[19] \backslash\{7,8,9,12,17\}$, the labelling of the vertices of the graph $G_{i}$ indicates how $\overline{G_{i}}$ may be contracted to $K_{7}^{-}$or, even, $K_{7}$. The vertices labelled $j \in[7]$ constitute the $j$ th branch set of a $K_{7}^{-}$minor or a $K_{7}$ minor. If the branch sets only constitute a $K_{7}^{-}$ minor, then it is because there is no edge between the branch sets of vertices labelled 1 and 7 , respectively.
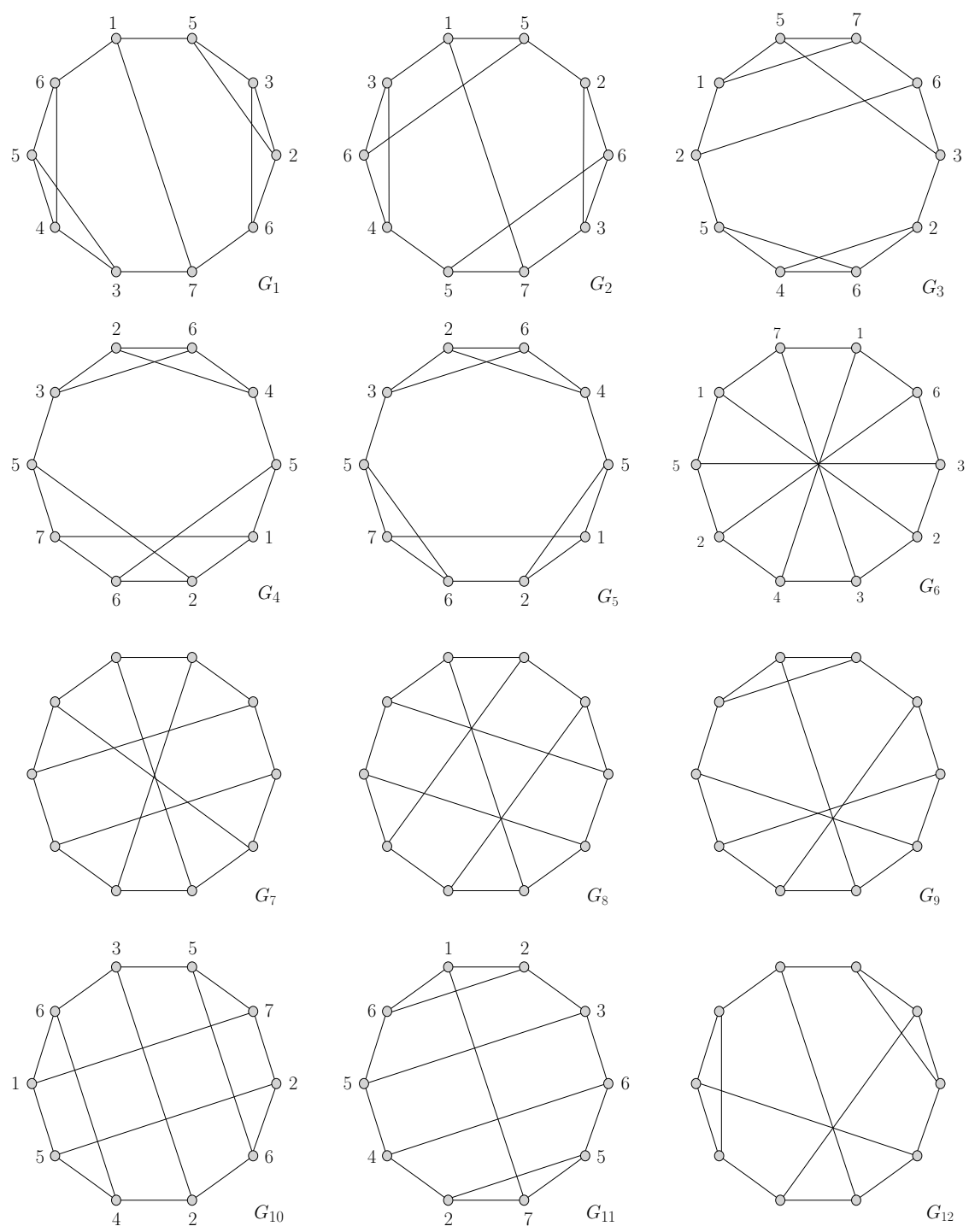

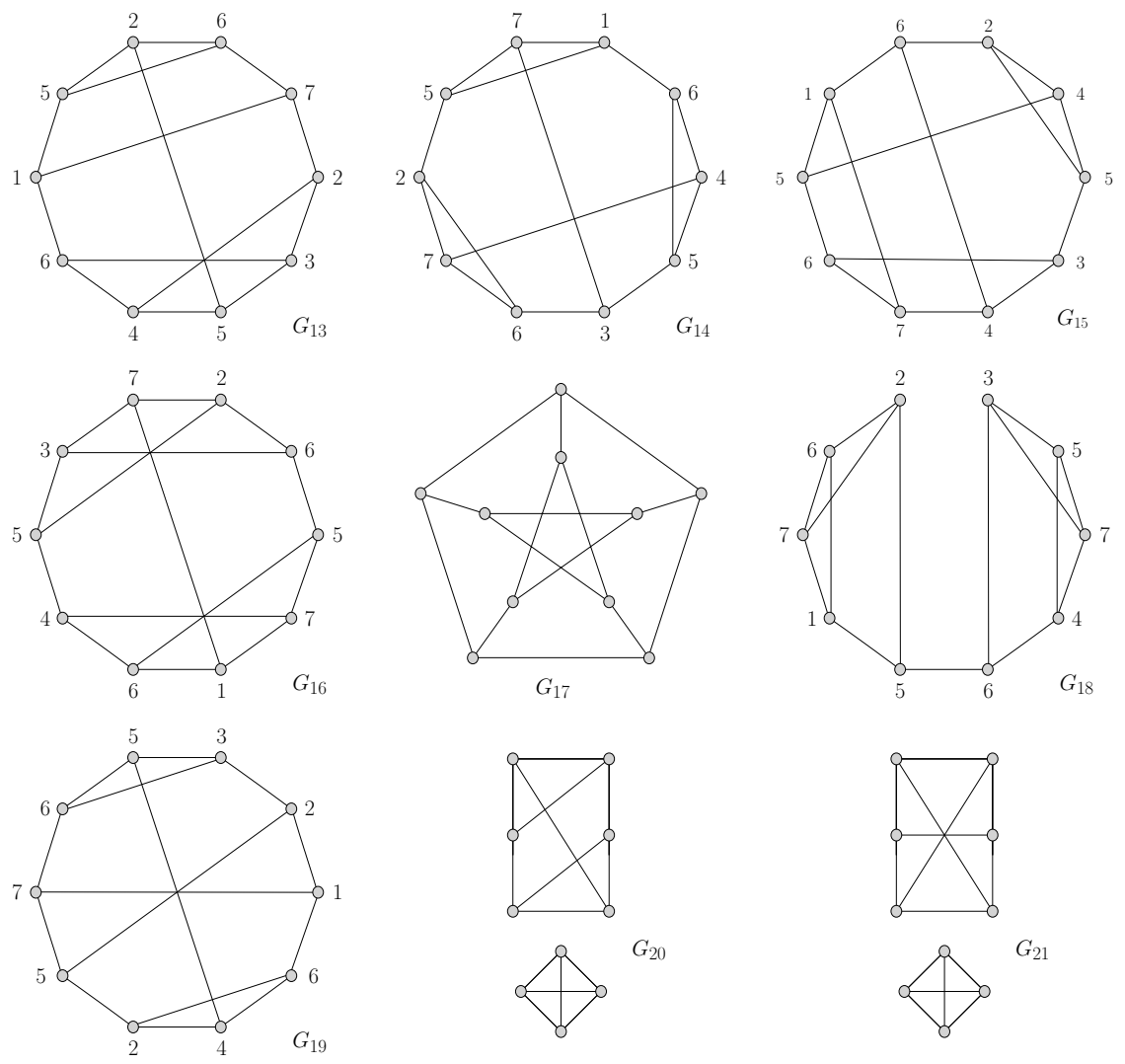

\section{Appendix B}

This section depicts 23 graphs $J_{i}(i \in[23])$. The vertices of each graph $J_{i}(i \in[23])$ are labelled with the integers 1 to 7 such that the vertices labelled $j \in[7]$ constitute the $j$ th branch set of a $K_{7}^{-}$minor or a $K_{7}$ minor. If the branch sets only constitute a $K_{7}^{-}$minor, then it is because there is no edge between the branch sets of vertices labelled 1 and 7 , respectively.
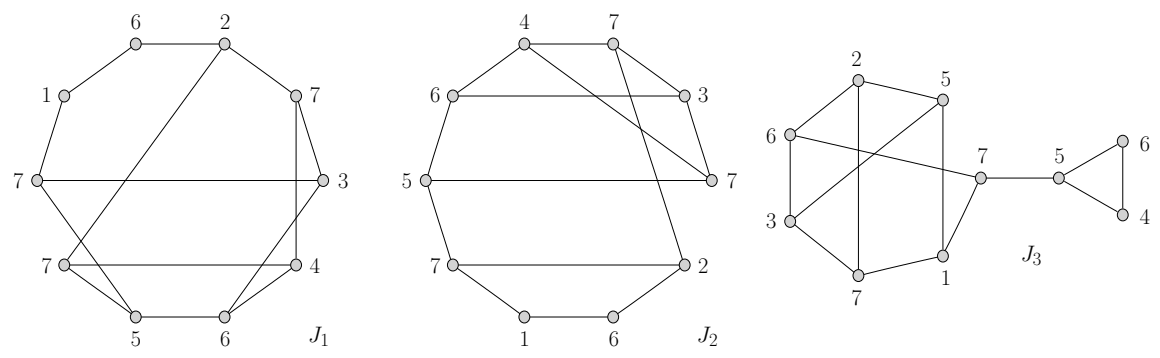

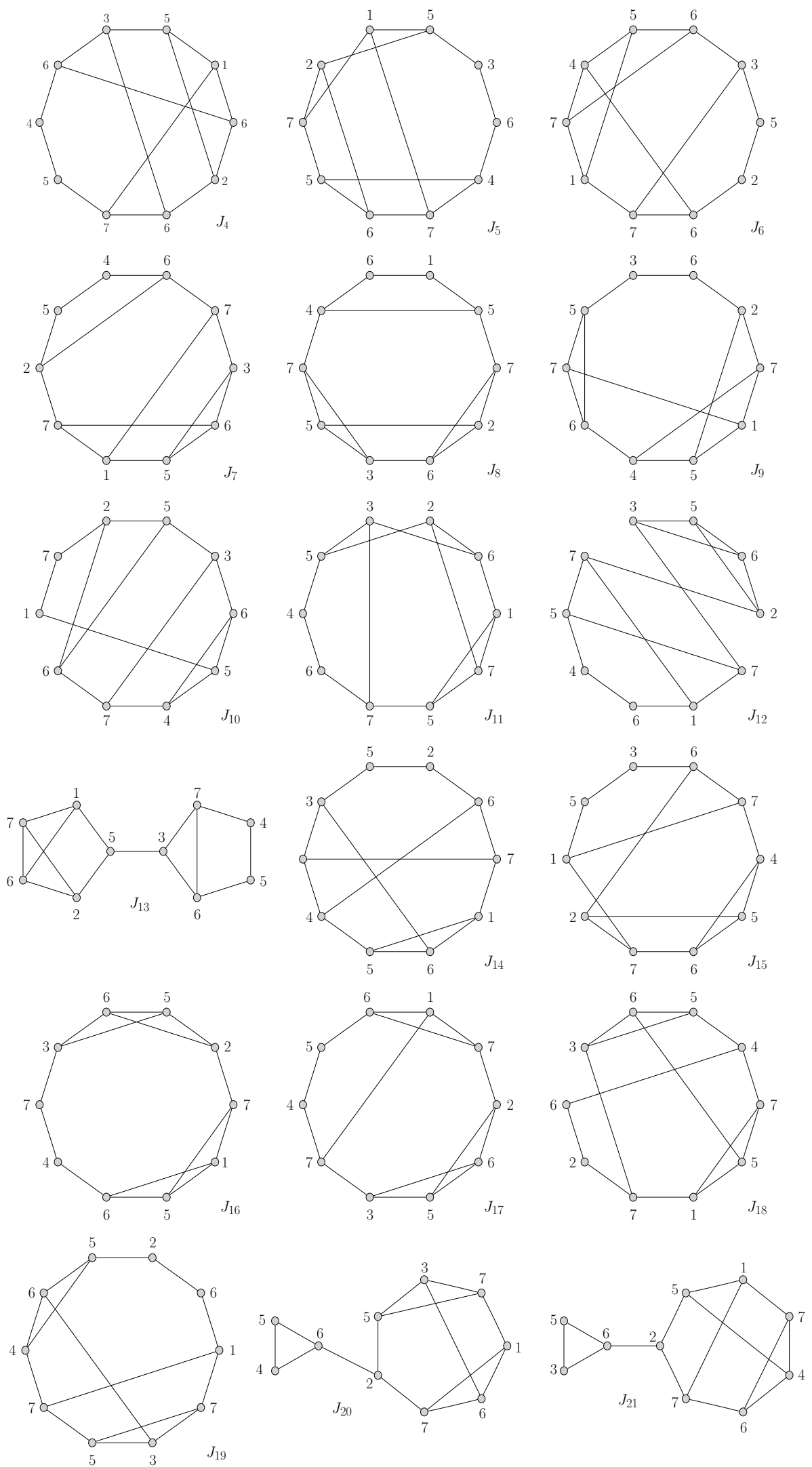

THE ELECTRONIC JOURNAL OF COMBINATORICS 18 (2011), \#P80 


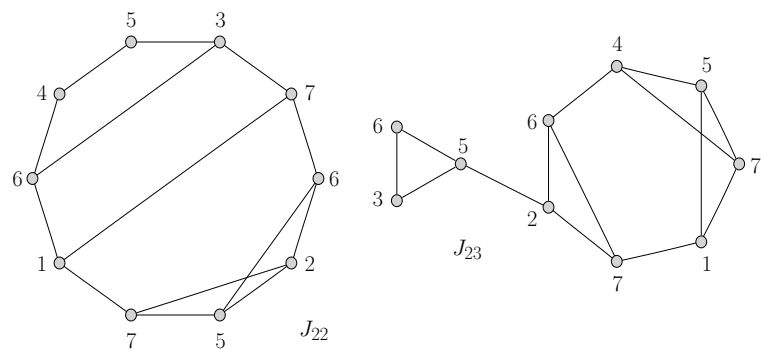

\section{References}

[1] K. Appel and W. Haken. Every planar map is four colorable. I. Discharging. Illinois J. Math., 21(3):429-490, 1977.

[2] K. Appel, W. Haken, and J. Koch. Every planar map is four colorable. II. Reducibility. Illinois J. Math., 21(3):491-567, 1977.

[3] B. Bollobás. Extremal graph theory, volume 11 of London Mathematical Society Monographs. Academic Press [Harcourt Brace Jovanovich Publishers], London, 1978.

[4] J. A. Bondy and U. S. R. Murty. Graph theory, volume 244 of Graduate Texts in Mathematics. Springer, New York, 2008.

[5] G. Chartrand, D. Geller, and S. Hedetniemi. Graphs with forbidden subgraphs. J. Combinatorial Theory Ser. B, 10:12-41, 1971.

[6] R. Diestel. Graph theory. 3rd revised and extended ed. Berlin: Springer, 2006.

[7] G. A. Dirac. A contraction theorem for abstract graphs. Math. Ann., 144:93-96, 1961.

[8] G. A. Dirac. On the structure of 5- and 6-chromatic abstract graphs. J. Reine Angew. Math., 214/215:43-52, 1964.

[9] P. Erdős. Problem 2. In Theory of Graphs (Proc. Colloq., Tihany, 1966), page 361. Academic Press, New York, 1968.

[10] H. Hadwiger. Über eine Klassifikation der Streckenkomplexe. Vierteljschr. Naturforsch. Ges. Zürich, 88:133-142, 1943.

[11] I. T. Jakobsen. On certain homomorphism-properties of graphs with applications to the conjecture of Hadwiger. Matematisk Institut, Aarhus Universitet, Aarhus, 1970. Doctoral dissertation, University of London, 1971, Various Publications Series, No. 15.

[12] I. T. Jakobsen. A homomorphism theorem with an application to the conjecture of Hadwiger. Studia Sci. Math. Hungar., 6:151-160, 1971.

[13] I. T. Jakobsen. On certain homomorphism properties of graphs. I. Math. Scand., 31: 379-404, 1972.

[14] T. R. Jensen and B. Toft. Graph coloring problems. Wiley-Interscience Series in Discrete Mathematics and Optimization. John Wiley \& Sons, New York, 1995. A Wiley-Interscience Publication. 
[15] L. K. Jørgensen. Contractions to $K_{8}$. J. Graph Theory, 18(5):431-448, 1994.

[16] K. Kawarabayashi and B. Toft. Any 7 -chromatic graph has $K_{7}$ or $K_{4,4}$ as a minor. Combinatorica, 25(3):327-353, 2005.

[17] K. Kawarabayashi, A. S. Pedersen, and B. Toft. Double-critical graphs and complete minors. Electron. J. Combin., 17(1):Research Paper 87, 27 pp., 2010.

[18] G. B. Khosrovshahi, Ch. Maysoori, and B. Tayfeh-Rezaie. A note on 3-factorizations of $K_{10}$. J. Combin. Des., 9(5):379-383, 2001.

[19] W. Mader. Homomorphiesätze für Graphen. Math. Ann., 178:154-168, 1968.

[20] W. Mader. $3 n-5$ edges do force a subdivision of $K_{5}$. Combinatorica, 18(4):569-595, 1998.

[21] B. McKay. The nauty page, 2009. URL http://cs.anu.edu.au/ bdm/nauty/.

[22] N. N. Mozhan. Twice critical graphs with chromatic number five. Metody Diskret. Analiz., (46):50-59, 73, 1987.

[23] R. C. Read and R. J. Wilson. An atlas of graphs. Oxford Science Publications. The Clarendon Press Oxford University Press, New York, 1998.

[24] N. Robertson, P. Seymour, and R. Thomas. Hadwiger's conjecture for $K_{6}$-free graphs. Combinatorica, 13(3):279-361, 1993.

[25] N. Robertson, D. Sanders, P. Seymour, and R. Thomas. The four-colour theorem. J. Combin. Theory Ser. B, 70(1):2-44, 1997.

[26] Z. Song. The extremal function for $K_{8}^{-}$minors. J. Combin. Theory Ser. B, 95(2): 300-317, 2005.

[27] M. Stiebitz. $K_{5}$ is the only double-critical 5-chromatic graph. Discrete Math., 64(1): 91-93, 1987.

[28] M. Stiebitz. On $k$-critical $n$-chromatic graphs. In Combinatorics (Eger, 1987), volume 52 of Colloq. Math. Soc. János Bolyai, pages 509-514. North-Holland, Amsterdam, 1988.

[29] B. Toft. Colouring, stable sets and perfect graphs. In Handbook of combinatorics, Vol. 1, 2, pages 233-288. Elsevier, Amsterdam, 1995.

[30] B. Toft. A survey of Hadwiger's conjecture. Congr. Numer., 115:249-283, 1996. Surveys in graph theory (San Francisco, CA, 1995).

[31] K. Wagner. Über eine Eigenschaft der ebenen Komplexe. Math. Ann., 114(1):570590, 1937.

[32] K. Wagner. Bemerkungen zu Hadwigers Vermutung. Math. Ann., 141:433-451, 1960.

[33] D. Woodall. Improper colourings of graphs. In Graph colourings (Milton Keynes, 1988), volume 218 of Pitman Res. Notes Math. Ser., pages 45-63. Longman Sci. Tech., Harlow, 1990. 